No. 570

May 2017

Numerical Estimation of Thermal Radiation Effects on Marangoni Convection of Dusty Fluid

S. Siddiqa, N. Begum,

Md. A. Hossain, A.A.A. Al-Rashed

ISSN: $\mathbf{2 1 9 0 - 1 7 6 7}$ 


\title{
Numerical Estimation of Thermal Radiation Effects on Marangoni Convection of Dusty Fluid
}

\author{
Sadia Siddiqa*,1, Naheed Begum ${ }^{\dagger}$, Md. Anwar Hossain ${ }^{\ddagger}$, Abdullah A.A.A. Al-Rashed ${ }^{\S}$ \\ * Department of Mathematics, COMSATS Institute of Information Technology, Kamra \\ Road, Attock, Pakistan \\ † Institute of Applied Mathematics (LSIII), TU Dortmund, Vogelpothsweg 87, Dortmund \\ D-44227, Germany \\ ${ }^{\ddagger}$ Fellow of Bangladesh Academy of Science, Professor (Rtd), University of Dhaka, Dhaka, \\ Bangladesh \\ $\S$ Dept. of Automotive and Marine Engineering Technology, College of Technological \\ Studies, The Public Authority for Applied Education and Training, Kuwait
}

\begin{abstract}
In this paper, numerical solutions to thermally radiating Marangoni convection of dusty fluid flow along a vertical wavy surface are established. The results are obtained with the understanding that the dust particles are of uniform size and dispersed in optically thick fluid. The numerical solutions of the dimensionless transformed equations are obtained through straightforward implicit finite difference scheme. In order to analyze the influence of various controlling parameters, results are displayed in the form of rate of heat transfer, skin friction coefficient, velocity and temperature profiles, streamlines and isotherms. It is observed that the variation in thermal radiation parameter significantly alters the corresponding particle pattern and extensively promotes the heat transfer rate.
\end{abstract}

\section{Nomenclature}

$\bar{a} \quad$ Dimensional amplitude of the vertical wavy surface $(m)$

a Dimensionless amplitude of the vertical wavy surface

$C_{f} \quad$ Skin friction coefficient

$c_{p} \quad$ Specific heat at constant pressure for fluid-phase $(\mathrm{J} / \mathrm{kgK})$

$c_{s} \quad$ Specific heat at constant pressure for particle-phase $(\mathrm{J} / \mathrm{kgK})$

$D_{\rho} \quad$ Mass concentration parameter

$g \quad$ Acceleration due to gravity $\left(\mathrm{m} / \mathrm{s}^{2}\right)$

$\hbar \quad$ Surface tension $(N / m)$

$\hbar_{m} \quad$ Reference surface tension $(N / m)$

$\hbar_{T} \quad$ Rate of change of surface tension with temperature $(N / m K)$

$G r_{L}$ Grashof number

$L \quad$ Characteristic length associated with wavy surface surface $(m)$

$M a \quad$ Marangoni number

$\mathrm{Nu}$ Nusselt number coefficient

$\mathrm{Pr} \quad$ Prandtl number

$\bar{p} \quad$ Dimensional pressure of carrier phase $\left(\mathrm{N} / \mathrm{m}^{2}\right)$

$\bar{p}_{p} \quad$ Dimensional pressure of particle phase $\left(\mathrm{N} / \mathrm{m}^{2}\right)$

$p \quad$ Dimensionless pressure of the carrier phase

\footnotetext{
${ }^{1}$ Corresponding author.

Email: saadiasiddiqa@gmail.com,Ph: +923335297152
} 
$p_{p} \quad$ Dimensionless pressure of the particle phase

Pr Prandtl number

$\overrightarrow{q_{r}} \quad$ Radiation heat flux $\left(W / m^{2}\right)$

$R_{d} \quad$ Thermal Radiation parameter

$T$ Dimensional temperature of fluid-phase $(K)$

$T_{\infty} \quad$ Ambient fluid temperature $(K)$

$T_{p} \quad$ Dimensional temperature of particle-phase $(K)$

$\bar{u}, \bar{v} \quad$ Dimensional fluid-phase velocity components $(\mathrm{m} / \mathrm{s})$

$\bar{u}_{p}, \bar{v}_{p}$ Dimensional particle-phase velocity components $(\mathrm{m} / \mathrm{s})$

$u, v \quad$ Dimensionless fluid-phase velocity components

$u_{p}, v_{p}$ Dimensionless particle-phase velocity components

$\bar{x}, \bar{y}$ Dimensional cartesian coordinates $(m)$

$x, y \quad$ Dimensionless coordinate system

\section{Greek letters}

$\alpha \quad$ Thermal diffusivity $\left(\mathrm{m}^{2} / \mathrm{s}\right)$

$\alpha_{r} \quad$ Rosseland mean absorption coefficient $(1 / m)$

$\alpha_{d} \quad$ Dusty fluid parameter

$\beta_{T} \quad$ Volumetric expansion coefficient $(1 / K)$

$\gamma \quad$ Ratio of $c_{p}$ to $c_{s}$

$\kappa \quad$ Thermal conductivity $(W / m K)$

$\theta \quad$ Dimensionless fluid-phase temperature

$\theta_{p} \quad$ Dimensionless particle-phase temperature

$\Theta_{w} \quad$ Surface temperature parameter

$\rho \quad$ Density of fluid-phase $\left(\mathrm{kg} / \mathrm{m}^{3}\right)$

$\rho_{p} \quad$ Density of particle-phase $\left(\mathrm{kg} / \mathrm{m}^{3}\right)$

$\lambda \quad$ Marangoni parameter

$\mu \quad$ Dynamic viscosity of fluid $(\mathrm{kg} / \mathrm{ms})$

$\nu \quad$ Kinematic viscosity of fluid $\left(\mathrm{m}^{2} / \mathrm{s}\right)$

$\tau_{m} \quad$ Velocity relaxation time of the particles $(s)$

$\tau_{T} \quad$ Thermal relaxation time of the particles $(s)$

$\tau_{w} \quad$ Shear stress at the surface

$Q_{w} \quad$ Rate of heat transfer at the surface

$\bar{\sigma}(\bar{x})$ Dimensional surface profile function $(m)$

$\sigma(x)$ Dimensionless surface profile function

$\sigma_{x} \quad$ First derivative of the function $\sigma$ w.r.t $x$

$\sigma_{x x}$ Second derivative of the function $\sigma$ w.r.t $x$

$\sigma_{s} \quad$ Scattering coefficient $(1 / m)$

$\sigma^{*} \quad$ Stephan-Boltzmann constant $\left(W / m^{2} K^{4}\right)$

\section{Subscripts}

$w \quad$ surface condition

$\infty \quad$ ambient condition

$p \quad$ particle phase

\section{Superscripts}




\section{Introduction}

The analysis of radiative heat transfer is significant in the framework of space and nuclear technology and numerous applications of thermal radiation can be found in chemical reaction phenomenon, storage of radioactive materials, chips in electronic devices, semiconductor wafers, magnetohydrodynamics electrical power generation, geophysics, nuclear power plants, various propulsion devices for aircraft and gas turbines. Numerous mathematical models have been discussed in literature in which thermally radiating heat flux either appears in the boundary conditions or sometimes in the energy equation. The inclusion of thermally radiative heat flux term in energy equation results in computational difficulties for solving the partial differential equations. However, some significant approximation, for instance, Rosseland diffusion approximation can be proposed to investigate the solution of governing equations with radiative heat transfer. Initially, the problem of radiative heat transfer together with convection and conduction was reported by Sparrow and Cess [1] and Özisik et al. [2]. Later on, Cess [3] investigated the thermal radiation heat transport in the boundary-layer region and obtained the solutions for system of nonlinear partial differential equations by incorporating the singular perturbation technique. Apraci [4] analyzed the non-equilibrium interaction between the laminar convective flow and thermal radiation. In later years, by utilizing the Rosseland diffusion approximation, numerous authors [5]-[7] discuss the free and/or mixed convective flows across/along the heated surfaces of various geometries, for example, flat plate (vertical/horizontal), rotating and non-rotating axisymmetric surfaces, sphere, channel and wavy surfaces under different boundary conditions.

The class of problems having Marangoni convection (or surface-tension-driven convection), is of major interest in biomedicine, industry and numerous practical applications like technology of coating flows, process of drying the semi-conductor wafers in microelectronics, film drainage in emulsions and foams, surfactant replacement therapy for neonatal infants and in various branches of space processing and micro-gravity. Napolitano [8]-[9] was the first who observed the existence of such dissipative layers in surface driven flows, which may usually be generated at the interface of two immiscible fluids. In this context, it was proved by Napolitano [10] that the governing set of equations in the bulk fluids have no explicit dependence on interface's geometry if arc length and the perpendicular distance to the interface are used as coordinates. However, this includes the average curvature of its dynamical and hydrostatical shapes and produces the kinematic, thermal and pressure couplings for the flow fields of two fluids. Afterwards, it was shown in [11] that, such coupling of the fields may be removed, when i) the viscosity ratio of the two fluids and ii) the momentum and thermal resistivity ratios of the two layers are strictly less than unity. Christopher and Wang [12] have investigated the influence of Prandtl number to visualize the relative change in thickness of boundary layers. Furthermore, the detailed numerical as well as analytical solutions for Marangoni boundary layers were discussed by several authors under various practical situations (for example see [13]-[17]).

The interest in studying the dynamics of heat transfer problems involving fluids 
containing spherical particles has mainly been increased in the past half century, because of its wide range of applications in many problems of engineering, physiological and atmospheric fields (see [18]). Initially, the analysis on gas-particulate suspension flow was done by [19]-[21] and after that numerous investigations were reported for the physical insight of contaminated flows (see Refs. [22]-[26]) under different physical circumstances. Particulate suspension flows have also been studied together with the interaction of irregular surfaces, which may promote rate of heat transfer extensively. In this regard, Siddiqa et al. [30]-[32] was the first to exploit the rough surfaces for the analysis of heat transfer in dusty fluid flow. In these papers, the author reported significant effects of surface non-uniformities for the natural convection of air and water particulate suspensions.

The interaction of thermal radiation on Marangoni convection flow of dusty fluid along a vertical wavy surface is not considered so far in the literature. Primitive variable formulation (PVF) is used for transforming the set of boundary layer equations of dusty fluid flow into a convenient system. Numerical solutions for the underlying coupled, nonlinear system is then obtained with the aid of implicit finite difference method together with the Thomas algorithm. In order to understand the particle behavior in the thermally radiating surface-tension driven flow, the results are presented graphically as coefficient of skin friction, rate of heat transfer, velocity and temperature profiles, streamlines and isotherms under the influence of important physical parameters.

\section{Mathematical Formulation}

In this paper, considerations has been given to 2D, incompressible, steady Marangoni convection of radiating dusty fluid along an heated vertical wavy surface, as shown in Fig. 1. The surface shape, $\bar{\sigma}(\bar{x})$, is described by the sinusoidal function of the form:

$$
\bar{y}_{w}=\bar{\sigma}(\bar{x})=\bar{a} \sin \left(\frac{2 \pi \bar{x}}{L}\right)
$$

where $\bar{a}$ is the dimensional amplitude of the wavy surface and $L$ the characteristic length associated with the irregular surface. The wavy surface is heated with an isothermal temperature $T_{w}$, which is higher than the ambient fluid temperature, $T_{\infty}$. Within the framework of this type of convection, a shear stress is developed in the interface, which is caused by the variation of surface tension. By taking into account the assumptions for two-phase dusty fluid flow presented in [23] and [24], the mathematical model is established as:

$$
\begin{gathered}
\frac{\partial \bar{u}}{\partial \bar{x}}+\frac{\partial \bar{v}}{\partial \bar{y}}=0 \\
\bar{u} \frac{\partial \bar{u}}{\partial \bar{x}}+\bar{v} \frac{\partial \bar{u}}{\partial \bar{y}}=-\frac{1}{\rho} \frac{\partial \bar{p}}{\partial \bar{x}}+\nu \nabla^{2} \bar{u}+g \beta_{T}\left(T-T_{\infty}\right)+\frac{\rho_{p}}{\rho \tau_{m}}\left(\bar{u}_{p}-\bar{u}\right) \\
\bar{u} \frac{\partial \bar{v}}{\partial \bar{x}}+\bar{v} \frac{\partial \bar{v}}{\partial \bar{y}}=-\frac{1}{\rho} \frac{\partial \bar{p}}{\partial \bar{y}}+\nu \nabla^{2} \bar{v}+\frac{\rho_{p}}{\rho \tau_{m}}\left(\bar{v}_{p}-\bar{v}\right) \\
\rho c_{p}\left(\bar{u} \frac{\partial T}{\partial \bar{x}}+\bar{v} \frac{\partial T}{\partial \bar{y}}\right)=\kappa \nabla^{2} T-\nabla \cdot \overrightarrow{q_{r}}+\frac{\rho_{p} c_{s}}{\tau_{T}}\left(T_{p}-T\right) \\
\frac{\partial \bar{u}_{p}}{\partial \bar{x}}+\frac{\partial \bar{v}_{p}}{\partial \bar{y}}=0
\end{gathered}
$$




$$
\begin{gathered}
\rho_{p}\left(\bar{u}_{p} \frac{\partial \bar{u}_{p}}{\partial \bar{x}}+\bar{v}_{p} \frac{\partial \bar{u}_{p}}{\partial \bar{y}}\right)=-\frac{\partial \bar{p}_{p}}{\partial \bar{x}}-\frac{\rho_{p}}{\tau_{m}}\left(\bar{u}_{p}-\bar{u}\right) \\
\rho_{p}\left(\bar{u}_{p} \frac{\partial \bar{v}_{p}}{\partial \bar{x}}+\bar{v}_{p} \frac{\partial \bar{v}_{p}}{\partial \bar{y}}\right)=-\frac{\partial \bar{p}_{p}}{\partial \bar{y}}-\frac{\rho_{p}}{\tau_{m}}\left(\bar{v}_{p}-\bar{v}\right) \\
\rho_{p} c_{s}\left(\bar{u}_{p} \frac{\partial T_{p}}{\partial \bar{x}}+\bar{v}_{p} \frac{\partial T_{p}}{\partial \bar{y}}\right)=-\frac{\rho_{p} c_{s}}{\tau_{T}}\left(T_{p}-T\right)
\end{gathered}
$$

where $(\bar{u}, \bar{v})$ are the velocity components in the $(\bar{x}, \bar{y})$ direction, $T$ the temperature, $\beta_{T}$ the volumetric expansion coefficient, $c_{p}$ the specific heat at constant pressure, $\kappa$ the thermal conductivity, $\rho$ the density and $\mu$ the kinematic viscosity of fluid phase. On the similar fashion, $\left(\bar{u}_{p}, \bar{v}_{p}\right), T_{p}, c_{s}$ and $\rho_{p}$ represent the the velocity components, temperature, specific heat and density for the particle phase, respectively. In addition, $g$ is the gravitational acceleration, $\tau_{m}$ the momentum relaxation time and $\tau_{T}$ the thermal relaxation time for dust particles. The radiative heat flux $\overrightarrow{q_{r}}$ in Eq. (5) is expressed by using Rosseland diffusion approximation and is given as:

$$
\overrightarrow{q_{r}}=-\frac{4 \sigma^{*}}{3 \kappa\left(\alpha_{r}+\sigma_{s}\right)} \nabla T^{4}
$$

where $\sigma^{*}, \sigma_{s}$ and $\alpha_{r}$ are the Stephan-Boltzmann constant, the scattering coefficient and Rosseland mean extinction coefficient, respectively. The physical model is to be solved under the following set of boundary conditions:

$$
\begin{gathered}
\mu \frac{\partial \bar{u}}{\partial \bar{y}}\left(\bar{x}, \bar{y}_{w}\right)=\hbar_{T} \frac{\partial T}{\partial \bar{x}}\left(\bar{x}, \bar{y}_{w}\right), \bar{v}\left(\bar{x}, \bar{y}_{w}\right)=0, T\left(\bar{x}, \bar{y}_{w}\right)-T_{\infty}=T_{0} \\
\bar{u}(\bar{x}, \infty)=0, T(\bar{x}, \infty)=T_{\infty} . \\
\mu \frac{\partial \bar{u}_{p}}{\partial \bar{y}}\left(\bar{x}, \bar{y}_{w}\right)=\hbar_{T} \frac{\partial T_{p}}{\partial \bar{x}}\left(\bar{x}, \bar{y}_{w}\right), \bar{v}_{p}\left(\bar{x}, \bar{y}_{w}\right)=0, T_{p}\left(\bar{x}, \bar{y}_{w}\right)-T_{\infty}=T_{0} \\
\bar{u}_{p}(\bar{x}, \infty)=0, T_{p}(\bar{x}, \infty)=T_{\infty} .
\end{gathered}
$$

where $\hbar_{T}=-\frac{\partial \hbar}{\partial T}$ is the rate of change of surface tension with temperature and $\hbar$ is the surface tension, which is assumed to be given by the linear relation:

$$
\hbar=\hbar_{m}-\hbar_{T}\left(T-T_{\infty}\right)
$$

$\hbar_{m}$ is the surface tension at a reference temperature $T_{\infty}$ and is assumed to be constant. The following variables are now introduced in Eqs. (1)-(13) in order to make the system dimensionless:

$$
\begin{aligned}
& \left(u, u_{p}\right)=\frac{\rho L}{\mu} G r_{L}^{-1 / 2}\left(\bar{u}, \bar{u}_{p}\right), \quad\left(v, v_{p}\right)=\frac{\rho L}{\mu} G r_{L}^{-1 / 4}\left(\left(\bar{v}, \bar{v}_{p}\right)-\sigma_{x}\left(\bar{u}, \bar{u}_{p}\right)\right), \quad x=\frac{\bar{x}}{L}, a=\frac{\bar{a}}{L}, \\
& y=\frac{\bar{y}-\bar{\sigma}(\bar{x})}{L} G r_{L}^{1 / 4}, \quad\left(\theta, \theta_{p}\right)=\frac{\left(T, T_{p}\right)-T_{\infty}}{T_{0}}, T_{0}=T_{w}-T_{\infty}, \quad\left(p, p_{p}\right)=\frac{L^{2}}{\rho \nu^{2} G r_{L}}\left(\bar{p}, \bar{p}_{p}\right), \\
& \sigma_{x}=\frac{d \bar{\sigma}}{d \bar{x}}=\frac{d \sigma}{d x}, \quad \sigma(x)=\frac{\bar{\sigma}(\bar{x})}{L}, \quad G r_{L}=\frac{g \beta_{T} T_{0} L^{3}}{\nu^{2}}, \quad \gamma=\frac{c_{s}}{c_{p}}, \quad \operatorname{Pr}=\frac{\nu}{\alpha}, \quad \Theta_{w}=\frac{T_{w}}{T_{\infty}}, \\
& R_{d}=\frac{4 \sigma^{*} T_{\infty}^{3}}{\kappa\left(\alpha_{r}+\sigma_{s}\right)}, \quad \lambda=\frac{M a}{G r_{L}^{3 / 4}}, \quad M a=\frac{\hbar_{T} T_{0} L}{\mu \nu}, \tau_{T}=\frac{3}{2} \gamma \tau_{m} \operatorname{Pr}, \quad D_{\rho}=\frac{\rho_{p}}{\rho}, \alpha_{d}=\frac{L^{2}}{\tau_{m} \nu G r_{L}^{1 / 2}}
\end{aligned}
$$


After introducing the above variables in Eqs. (1)-(13), we get:

$$
\begin{gathered}
\frac{\partial u}{\partial x}+\frac{\partial v}{\partial y}=0 \\
u \frac{\partial u}{\partial x}+v \frac{\partial u}{\partial y}=-\frac{\partial p}{\partial x}+\sigma_{x} G r_{L}^{1 / 4} \frac{\partial p}{\partial y}+\left(1+\sigma_{x}^{2}\right) \frac{\partial^{2} u}{\partial y^{2}}+\theta+D_{\rho} \alpha_{d}\left(u_{p}-u\right) \\
\sigma_{x}\left(u \frac{\partial u}{\partial x}+v \frac{\partial u}{\partial y}\right)+\sigma_{x x} u^{2}=-G r_{L}^{1 / 4} \frac{\partial p}{\partial y}+\sigma_{x}\left(1+\sigma_{x}^{2}\right) \frac{\partial^{2} u}{\partial y^{2}}+\sigma_{x} D_{\rho} \alpha_{d}\left(u_{p}-u\right) \\
u \frac{\partial \theta}{\partial x}+v \frac{\partial \theta}{\partial y}=\frac{\left(1+\sigma_{x}^{2}\right)}{\operatorname{Pr}}\left[\frac{\partial^{2} \theta}{\partial y^{2}}+\frac{\partial}{\partial y}\left(\left[\frac{4}{3} R_{d}\left(1+\left(\Theta_{w}-1\right) \theta\right)^{3}\right] \frac{\partial \theta}{\partial y}\right)\right]+\frac{2}{3 \operatorname{Pr}} D_{\rho} \alpha_{d}\left(\theta_{p}-\theta\right) \\
u_{p} \frac{\partial u_{p}}{\partial x}+v_{p} \frac{\partial u_{p}}{\partial y}=-\frac{\partial v_{p}}{\partial x}+\sigma_{x} G r_{L}^{1 / 4} \frac{\partial p_{p}}{\partial y}-\alpha_{d}\left(u_{p}-u\right) \\
\sigma_{x}\left(u_{p} \frac{\partial u_{p}}{\partial x}+v_{p} \frac{\partial u_{p}}{\partial y}\right)+u_{p}^{2} \sigma_{x x}=-G r_{L}^{1 / 4} \frac{\partial p_{p}}{\partial y}-\alpha_{d} \sigma_{x}\left(u_{p}-u\right) \\
u_{p} \frac{\partial \theta_{p}}{\partial x}+v_{p} \frac{\partial \theta_{p}}{\partial y}=-\frac{2}{3 \gamma \operatorname{Pr}} \alpha_{d}\left(\theta_{p}-\theta\right)
\end{gathered}
$$

The transformed boundary conditions are:

$$
\begin{gathered}
\frac{\partial u}{\partial y}(x, 0)=-\lambda \frac{\partial \theta}{\partial x}(x, 0), v(x, 0)=0, \theta(x, 0)=1 \\
u(x, \infty)=0, \theta(x, \infty)=0 \\
\frac{\partial u_{p}}{\partial y}(x, 0)=-\lambda \frac{\partial \theta_{p}}{\partial x}(x, 0), v_{p}(x, 0)=0, \theta_{p}(x, 0)=1 \\
u_{p}(x, \infty)=0, \theta_{p}(x, \infty)=0
\end{gathered}
$$

The terms $G r_{L}^{1 / 4} \partial p / \partial y$ and $G r_{L}^{1 / 4} \partial p_{p} / \partial y$ in Eqs. (16) and (20) depicts the fact that the order of pressure gradient along the $y$ direction is $O\left(G r_{L}^{-1 / 4}\right)$. This implies that the lowest order $x$ directional pressure gradient can be calculated from the inviscid-flow solution. As there is no externally induced free stream in the present problem, therefore, this pressure gradient is zero. Therefore, eliminating the term $\partial p / \partial y$ from Eqs. (16) and (17), one gets:

$$
u \frac{\partial u}{\partial x}+v \frac{\partial u}{\partial y}+\frac{\sigma_{x} \sigma_{x x}}{\left(1+\sigma_{x}^{2}\right)} u^{2}=\left(1+\sigma_{x}^{2}\right) \frac{\partial^{2} u}{\partial y^{2}}+\frac{\theta}{\left(1+\sigma_{x}^{2}\right)}+D_{\rho} \alpha_{d}\left(u_{p}-u\right)
$$

Similarly, by removing $\partial p_{p} / \partial y$ from Eqs. (20) and (21), we have:

$$
u_{p} \frac{\partial u_{p}}{\partial x}+v_{p} \frac{\partial u_{p}}{\partial y}+\frac{\sigma_{x} \sigma_{x x}}{\left(1+\sigma_{x}^{2}\right)} u_{p}^{2}=-D_{\rho} \alpha_{d}\left(u_{p}-u\right)
$$

Numerical solutions of the above problem are obtained after transforming the above system of equations into the following form:

$$
\frac{1}{2} U+X \frac{\partial U}{\partial X}-\frac{1}{4} Y \frac{\partial U}{\partial Y}+\frac{\partial V}{\partial Y}=0
$$




$$
\begin{aligned}
& \left(\frac{1}{2}+\frac{X \sigma_{X} \sigma_{X X}}{\left(1+\sigma_{X}^{2}\right)}\right) U^{2}+X U \frac{\partial U}{\partial X}+\left(V-\frac{1}{4} Y U\right) \frac{\partial U}{\partial Y}=\left(1+\sigma_{X}^{2}\right) \frac{\partial^{2} U}{\partial Y^{2}}+\frac{\Theta}{\left(1+\sigma_{X}^{2}\right)} \\
& +D_{\rho} \alpha_{d} X^{1 / 2}\left(U_{p}-U\right) \\
& X U \frac{\partial \Theta}{\partial X}+\left(V-\frac{1}{4} Y U\right) \frac{\partial \Theta}{\partial Y}=\frac{\left(1+\sigma_{X}^{2}\right)}{\operatorname{Pr}} \frac{\partial}{\partial Y}\left[\left(1+\frac{4}{3} R_{d}\left(1+\left(\Theta_{w}-1\right) \Theta\right)^{3}\right) \frac{\partial \Theta}{\partial Y}\right] \\
& +\frac{2}{3 \operatorname{Pr}} D_{\rho} \alpha_{d} X^{1 / 2}\left(\Theta_{p}-\Theta\right) \\
& \frac{1}{2} U_{p}+X \frac{\partial U_{p}}{\partial X}-\frac{1}{4} Y \frac{\partial U_{p}}{\partial Y}+\frac{\partial V_{p}}{\partial Y}=0 \\
& \left(\frac{1}{2}+\frac{X \sigma_{X} \sigma_{X X}}{\left(1+\sigma_{X}^{2}\right)}\right) U_{p}^{2}+X U_{p} \frac{\partial U_{p}}{\partial X}+\left(V_{p}-\frac{1}{4} Y U_{p}\right) \frac{\partial U_{p}}{\partial Y}=-\alpha_{d} X^{1 / 2}\left(U_{p}-U\right) \\
& X U_{p} \frac{\partial \Theta_{p}}{\partial X}+\left(V_{p}-\frac{1}{4} Y U_{p}\right) \frac{\partial \Theta_{p}}{\partial Y}=-\frac{2}{3 \gamma \operatorname{Pr}} \alpha_{d} X^{1 / 2}\left(\Theta_{p}-\Theta\right) \\
& \frac{\partial U}{\partial Y}(X, 0)=-\lambda X^{-1 / 4} \frac{\partial \Theta}{\partial X}(X, 0), V(X, 0)=0, \Theta(X, 0)=1 \\
& U(X, \infty)=0, \Theta(X, \infty)=0 \text {. } \\
& \frac{\partial U_{p}}{\partial Y}(X, 0)=-\lambda X^{-1 / 4} \frac{\partial \Theta_{p}}{\partial X}(X, 0), V_{p}(X, 0)=0, \Theta_{p}(X, 0)=1 \\
& U_{p}(X, \infty)=0, \Theta_{p}(X, \infty)=0 \text {. }
\end{aligned}
$$

where

$$
x=X, \quad y=x^{\frac{1}{4}} Y, \quad\left(u, u_{p}\right)=x^{\frac{1}{2}}\left(U, U_{p}\right), \quad\left(v, v_{p}\right)=x^{-\frac{1}{4}}\left(V, V_{p}\right), \quad\left(\theta, \theta_{p}\right)=\left(\Theta, \Theta_{p}\right)
$$

In order to solve the non-linear system of partial differential equations (27)-(34) implicit finite difference method is employed. The discretization procedure and numerical scheme is carried out by considering the details given in [7]. After determining all the unknown of the system, the dimensionless expressions for the physical quantities of interest like skin friction coefficient $\tau_{w}$ and heat transfer rate $Q_{w}$ are obtained as:

$$
\begin{aligned}
\tau_{w} & =C_{f}\left(\frac{G r_{L}^{-3}}{X}\right)^{1 / 4}=\sqrt{1+\sigma_{X}^{2}}\left(\frac{\partial U}{\partial Y}\right)_{Y=0} \\
Q_{w}=N u\left(\frac{G r_{L}}{X}\right)^{-1 / 4} & =-\sqrt{1+\sigma_{X}^{2}}\left(1+\frac{4}{3} R_{d} \Theta_{w}^{3}\right)\left(\frac{\partial \Theta}{\partial Y}\right)_{Y=0}
\end{aligned}
$$

In the upcoming section, the numerical results are graphed and discussed.

\section{Results and Discussion}

The prime purpose of present study is to analyze the Marangoni convection boundary-layer flow of a two-phase dusty fluid. In the analysis, contaminated fluid flows along a vertical wavy surface and Rosseland diffusion approximation is used to elucidate the effects of thermal radiation. The two-dimensional simulations are performed for non-linear coupled system by employing the implicit finite difference method. The computed results are reported for the overall effectiveness of presence of thermal radiation and mass concentration of dust particles in a surface-tension driven convection. The solutions are presented to 
record the influence of radiation parameter, $R_{d}$, mass concentration parameter, $D_{\rho}$, surface temperature parameter, $\Theta_{w}$ and the amplitude of wavy surface parameter, $a$, on rate of heat transfer and skin friction coefficient, velocity and temperature profiles and streamlines and isotherms. Specifically, the results are established for the contaminated water i.e, $\operatorname{Pr}=7.0$, $D_{\rho}=10.0$ and $\gamma=0.1$ and these particular parametric values for dusty water are taken from study of Apazidis [33], whereas the overall range of the other parameters is set as: $R_{d}=0.0,1.0,2.0,3.0, \Theta_{w}=1.0,1.1,1.2,1.3, \alpha_{d}=0.1, \lambda=1.0$ and $a=0.2,0.3,0.4,0.5$.

The effect of thermal radiation parameter is entered in Table 1 for the quantities $\tau_{w}$ and $Q_{w}$. The quantitative data clearly shows much influence of thermal radiation parameter, $R_{d}$, on the rate of heat transfer as compared to the skin friction coefficient. Particularly, the values of $Q_{w}$ indicates that $R_{d}$ participates sufficiently in increasing the rate of heat transfer near the leading edge.

Table 1: Numerical values of $\tau_{w}$ and $Q_{w}$ for $R_{d}=0.0,2.0$, while $D_{\rho}=5.0, \operatorname{Pr}=7.0, \gamma=0.1$, $\alpha_{d}=0.1, \lambda=1.0, \Theta_{w}=1.1$ and $a=0.3$.

\begin{tabular}{|c|c|c|c|c|}
\hline \multirow{2}{*}{$X$} & \multicolumn{2}{|c|}{$\tau_{w}$} & \multicolumn{2}{c|}{$Q_{w}$} \\
\cline { 2 - 5 } & $R_{d}=0.0$ & $R_{d}=2.0$ & $R_{d}=0.0$ & $R_{d}=2.0$ \\
\hline 0.01999 & 0.00162 & 0.00134 & 0.90981 & 2.06639 \\
1.00900 & 0.00162 & 0.00140 & 0.93683 & 2.15625 \\
3.00700 & 0.00177 & 0.00152 & 0.93366 & 2.12870 \\
5.00500 & 0.00190 & 0.00166 & 0.92337 & 2.11096 \\
7.00300 & 0.00203 & 0.00183 & 0.91330 & 2.10182 \\
9.00100 & 0.00218 & 0.00203 & 0.90425 & 2.09792 \\
\hline
\end{tabular}

In order to ensure the accuracy of our scheme and computational results, a comparison is also being done with the available published data. It should be noted that the numerical results obtained herein reduce to those reported by Yao [27] for sinusoidal wavy surface provided that the radiation effects and thermal Marangoni convection is ignored. In [27], Keller box method is used to obtain solutions over the whole range of axial coordinate $\xi$, while the present computational results are obtained from implicit finite difference method. Still the solutions can be compared graphically for heat transfer coefficient by keeping the physical parameters as: $\operatorname{Pr}=1.0, a=0.1,0.3$ for pure fluid in the absence of radiation and Marangoni convection effects. Therefore, the comparison shown quite an excellent agreement between the two studies and this also validates our numerical scheme.

The effect of mass concentration parameter $D_{\rho}$ on $\tau_{w}$ and $Q_{w}$ is presented in Fig. 3 . For the purpose of comparison, the skin friction coefficient and the rate of heat transfer are also plotted for pure fluid i.e. water without inert particles. It is observed from Fig. 3 that both the skin friction coefficient and rate of heat transfer are minimum for non-zero values of the mass concentration parameter $D_{\rho}$. Specifically, the effect of $D_{\rho}$ is more pronounced for $Q_{w}$ and reduces considerable owing to an increase in the values of $D_{\rho}$. This may happen due to the collision of dispersed particles which resist the flow they can exchange momentum and energy. As a result the velocity of contaminated fluid reduces as compared to the pure fluid. Further, the shear stress at the wall will be correspondingly smaller.

Contribution of thermal radiation parameter, $R_{d}$, on $\tau_{w}$ and $Q_{w}$ is depicted in Fig. 4. The variation in skin friction is given in Fig. 4(a), which shows the reduction in magnitude 
due to the increase in thermal radiation parameter $R_{d}$. Therefore, skin friction is maximum for $R_{d}=0.0$ and decreases when $R_{d}$ is increased from 0.0 to 3.0. The amplitude of the curves representing the $\tau_{w}$ also reduces when $R_{d}$ grows. Like the typical behavior of thermal radiation, it is observed that the rate of heat transfer increases significantly when thermal radiation is increased. Fig. 4(b) reveals the fact that the rate of heat transfer is very low when the fluid is non-radiating (i.e, when $R_{d}=0.0$ ) and promoted extensively when radiation parameter is penetrated into the mechanism. This is expected because $R_{d}$ raised the temperature of the dusty fluid in the boundary-layer region. In the present situation, it can be concluded that the thermal radiation acts as a heat source and large values of $R_{d}$ contributes in magnifying the amplitude of the waves representing $Q_{w}$. Further, in case of heat transfer rate, the shape of the wavy surface also changes for non-zero values of $R_{d}$.

Figure 5 is presenting the variation in $\tau_{w}$ and $Q_{w}$ by varying the values of surface temperature parameter, $\Theta_{w}$, for the flow of the contaminated water. The surface temperature parameter do not contributes anything in skin friction coefficient as it shows a uniform behavior for all $\Theta_{w}$ while on the other hand the rate of heat transfer shows a remarkable increase within the boundary layer region when $\Theta_{w}$ increases. It is important to mention here that $\Theta_{w}=1.0$ corresponds to the case when the fluid will be non-radiating. It can be seen from Fig. 5(b) that the rate of heat transfer is very low for $\Theta_{w}=1.0$ and is boosted up when $\Theta_{w}>>1$.0. The reason for such behavior is similar to the previous result. That is, temperature of the fluid increases sufficiently and as a result the fluid accelerates more rapidly in the boundary-layer regime. Moreover, the shape of the curves also tends to change as soon as fluid moves in the stream-wise direction.

The influence of thermal radiation parameter $R_{d}$ is also shown on the velocity and temperature profiles in Fig. 6 for the carrier phase and the particle phase. It is seen that the thermal radiation parameter $R_{d}$ participates significantly in increasing the velocity profiles for both phases (see Fig. 6(a)). It is interesting to observe that the velocity profiles for $R_{d}=0.0$ quickly acquires the asymptotical state in the momentum boundary layer region. In addition, similar behavior is recorded in the distribution of temperature profiles in Fig. 6(b). The thermal and viscous layers grow sufficiently by magnifying the values of conduction-radiation parameter, $R_{d}$. Thus, it can be concluded from this study that the non-zero values of thermal radiation parameter $R_{d}$ increases the thermal as well as momentum boundary layer thickness.

The effect of mass concentration of dust particles parameter, $D_{\rho}$ on streamlines and isotherms for water-particle mixture is shown in Fig. 7. It is interesting to see that by loading the dust particles the velocity and the temperature distribution of carrier fluid increases considerably when $D_{\rho}$ increases. When particles are loaded in the fluid at relatively high ratio, i.e, $D_{\rho}=10.0$, the momentum and energy is exchanged with the base fluid and particles collide as well to increases the overall velocity and temperature within the boundary-layer region. It is further noted that the amplitude of the waves also increases at the outer edge of the boundary layer.

\section{Conclusion}

In this article, we have studied the interaction of thermal radiation on Marangoni convection of two phase dusty fluid. The water particulate mixture is considered to flows along the semi-infinite vertical wavy surface. Coordinate transformations (primitive variable for- 
mulations) are applied to switch the governing equations of the carrier and the dispersed phase into another set of equations, which are then integrated by finite difference method. Influence of various emerging parameters are explored by expressing their relevance on skin friction coefficient and rate of heat transfer. Velocity distribution, temperature distribution, streamlines and isotherms are also plotted to visualize the flow behavior. From this analysis, it is concluded that increase in the values of radiation parameter or surface temperature parameter leads to an increase in the rate of heat transfer, velocity distribution and the temperature distribution.

\section{References}

[1] Sparrow, E. M., Cess, R. D., Radiation heat transfer, augmented edition, hemisphere Media, Int. J. Heat Mass Transfer, 5, 1962, 179-806.

[2] Özisik, M. N., Radiative transfer and interactions with conduction and convection, 1973 (John Wiley \& Sons, NewYork).

[3] Cess, R. D., The interaction of thermal radiation in boundary-layer heat transfer, Proceeding of 3rd International Heat Transfer Conference, bf5, 1966, 154-163.

[4] Arpaci, V. S., Effect of thermal radiation on the laminar free convection from a heated vertical plate, Int. J. Heat Mass Transfer, 11, 1968, 871-881.

[5] Molla, M. M., Hossain, M. A., Radiation effect on mixed convection laminar flow along a vertical wavy surface, Int. J. Therm. Sci., 46, 2007, 926-935.

[6] Siddiqa, S., Faryad, A., Begum, N., Hossain, M. A., Gorla, R. S. R., Periodic magnetohydrodynamic natural convection flow of a micropolar fluid with radiation, Int. J. Therm. Sci., 111, 2017, 215-222.

[7] Siddiqa, S., Begum, N., Hossain, M. A., Radiation effects from an isothermal vertical wavy cone with variable fluid properties, Appl. Math. Comput., 289, 2016, 149-158.

[8] Napolitano, L. G., Microgravity fluid dynamics, In:2nd Levitch conference, Washington, 1978.

[9] Napolitano, L. G., Marangoni boundary layers, In: Proceedings of the 3rd European symposium on material science in space, Grenoble, ESA SP-142, 1979.

[10] Napolitano, L. G., Surface and buoyancy driven free convection, Acta Astronautica, 9, 1982, 199215.

[11] Napolitano, L. G., Golia, C., Coupled Marangoni boundary layers, Acta Astronautica, 8, 1981, 417-434.

[12] Christopher, D. M., Wang, B., Prandtl number effects for Marangoni convection over a flat surface, Int. J. Therm. Sci., 40, 2001, 564-570.

[13] Golia, C., Viviani, A., Non isobaric boundary layers related to Marangoni flows, Meccanica, 21, 1986, 200-204. 
[14] Pop, I., Postelnicu, A., Grosan, T., Thermosolutal Marangoni forced convection boundary layers, Meccanica, 36, 2001, 555-571.

[15] Magyari, E., Chamkha, A. J., Exact analytical solutions for thermosolutal Marangoni convection in the presence of heat and mass generation or consumption, Heat and Mass Transfer, 43, 2007, 965-974.

[16] Magyari, E. Chamkha, A. J., Exact analytical results for the thermosolutal MHD Marangoni boundary layer, Int. J. Therm. Sci., 47, 2008, 848-857.

[17] Zueco, J., Bèg, O. A., Network numerical simulation of hydromagnetic Marangoni mixed convection boundary layers, Chemical Eng. Commun., 198, 2011, 552-571.

[18] Rudinger, G., Fundamentals of gas-particle flow, Elsevier Scientific Publishing Co., Amsterdam, 1980.

[19] Farbar, L., Morley, M. J., Heat transfer to flowing gas-solid mixtures in a circular tube, Ind. Eng. Chem., 49, 1957, 1143-1150.

[20] Marble, F. E., Dynamics of a gas containing small solid particles, combustion and propulsion, 5th AGARD colloquium, Pergamon press, 1963.

[21] Singleton, R. E., Fluid mechanics of gas-solid particle flow in boundary layers, Ph.D. Thesis, California Institute of Technology, 1964.

[22] Michael, D. H., Miller, D. A., Plane parallel flow of a dusty gas, Mathematica, 13, 1966, 97-109.

[23] Saffman, P. G., On the stability of laminar flow of a dusty gas, J. Fluid Mech., 13, $1962,120-128$.

[24] Siddiqa, S., Hossain, M. A., Saha, S. C., Two-phase natural convection flow of a dusty fluid, Int. J. Numer. Method, 25, 2015, 1542-1556.

[25] Begum, N., Siddiqa, S., Hossain, M. A., Gorla, R. S. R., Natural convection flow of two-phase dusty gas with variable therophysical properties, Journal of Thermophysics and Heat Transfer, 2017, DOI: 10.2514/1.T5057.

[26] Siddiqa, S., Begum, N., Hossain, M. A., Mustafa, N., Gorla, R. S. R., Two-phase dusty fluid flow along a cone with variable fluid properties, , Heat Mass Transfer, 2017, DOI 10.1007/s00231-016-1918-y.

[27] Yao, L. S., Natural convection along a vertical wavy surface, J. Heat Transfer, 105, 1983, 465-468.

[28] Hossain, M. A., Rees, D. A. S., Combined heat and mass transfer in natural convection flow from a vertical wavy surface, Acta Mechanica, 136, 1999, 133-141.

[29] Siddiqa, S., Hina, G., Begum, N., Hossain, M. A., Gorla, R. S. R., Numerical solutions of nanofluid bioconvection due to gyrotactic microorganisms along a vertical wavy cone, Int. J. Heat Mass Transfer, 101, 2016, 608-613. 
[30] Siddiqa, S., Begum, N., Hossain, M. A., Compressible dusty gas along a vertical wavy surface, Appl. Math. Comput., 293, 2017, 600-610.

[31] Siddiqa, S., Begum, N., Hossain, M. A., Gorla, R. S. R., Numerical solutions of natural convection flow of a dusty nanofluid about a vertical wavy truncated cone, Journal of Heat Transfer, 139, 2017, DOI: 10.1115/1.4034815.

[32] Siddiqa, S., Begum, N., Hossain, M. A., Massarotti, N., Influence of thermal radiation on contaminated air and water flow past a vertical wavy frustum of a cone, Int. Commun. Heat Mass, 76, 2016, 63-68.

[33] Apazidis, N., Temperature distribution and heat transfer in a particle-fluid flow past a heated horizontal plate, Int. J. Multiphase Flow, 16, 1990, 495-513. 


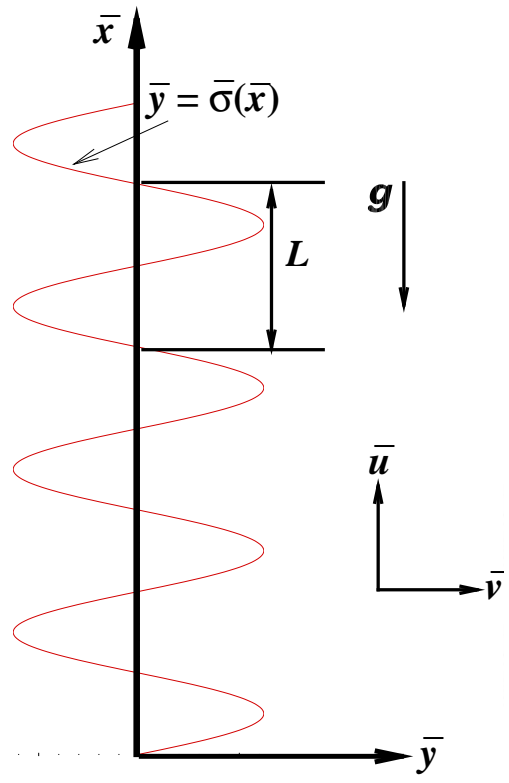

Fig. 1 The physical model. 


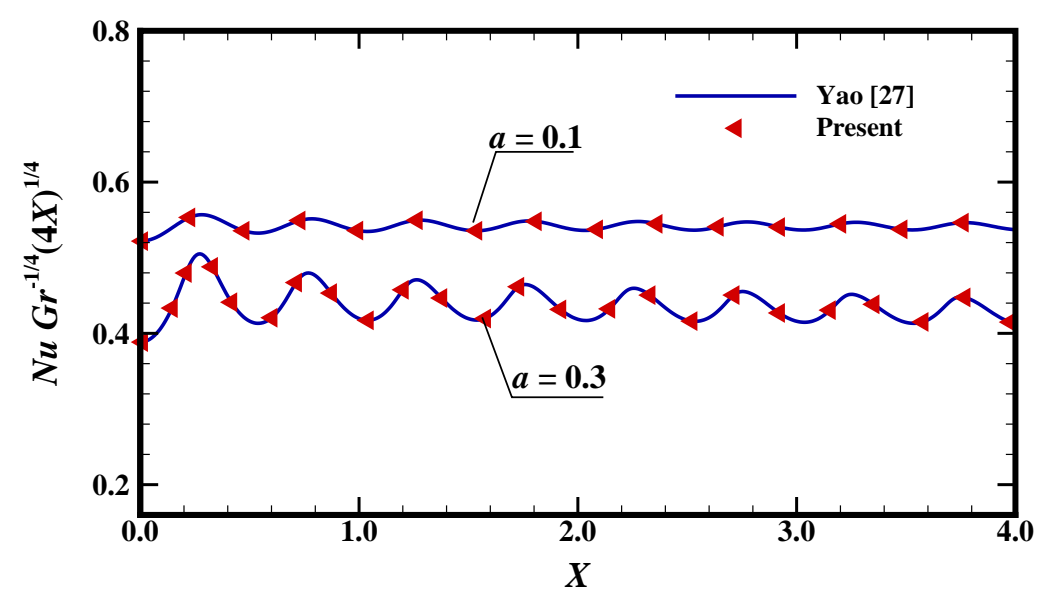

Fig. 2 Local Nusselt number coefficient for $a=0.1,0.3$, while $\operatorname{Pr}=1.0, \Theta_{w}=1.1$ and $\lambda=R_{d}=D_{\rho}=\alpha_{d}=0.0$.
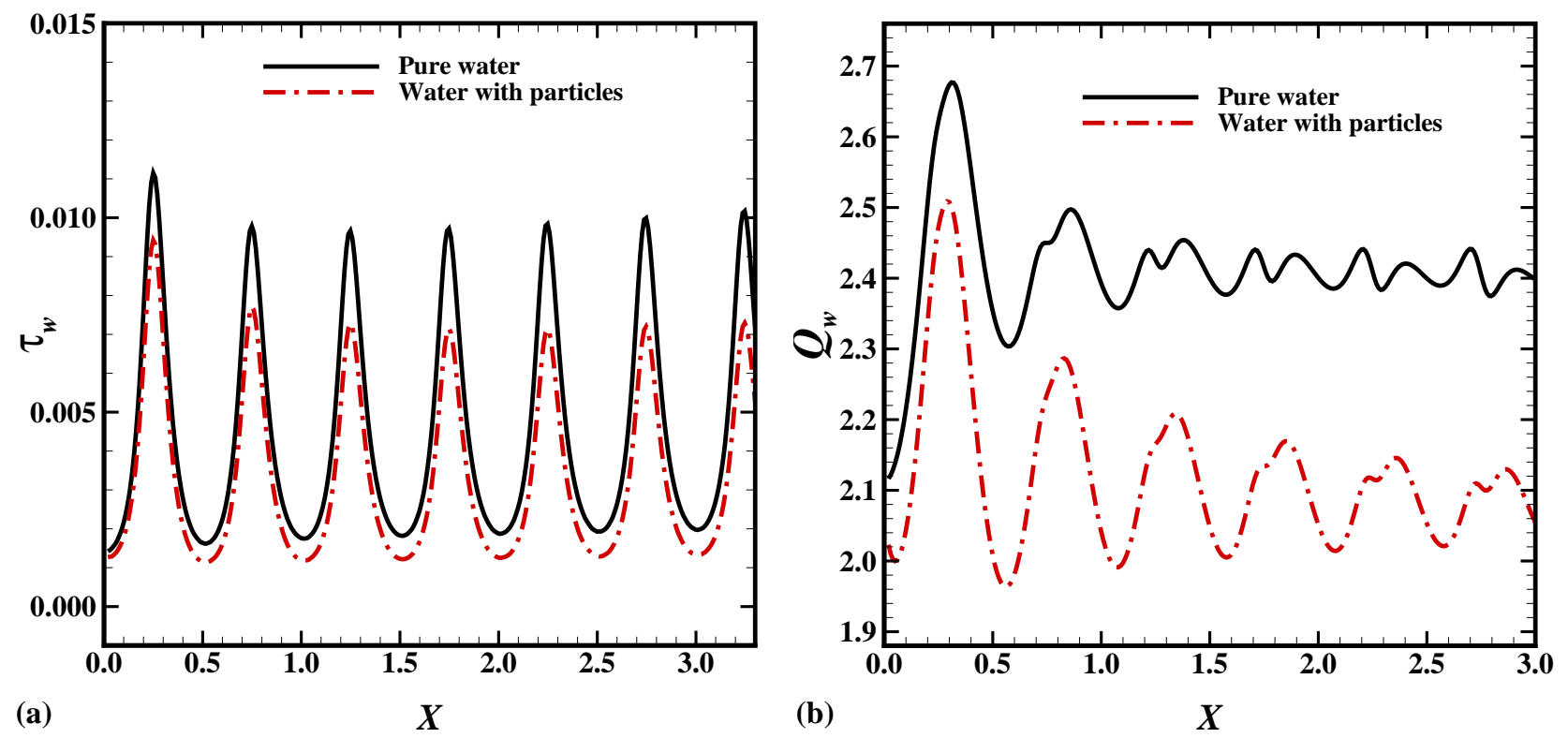

Fig. 3(a) Skin friction and (b) Rate of heat transfer for $D_{\rho}=0.0,10.0$ while $\operatorname{Pr}=7.0, \gamma=0.1, \alpha_{d}=0.1, \lambda=1.0, R_{d}=2.0, \Theta_{w}=1.1$ and $a=0.3$. 

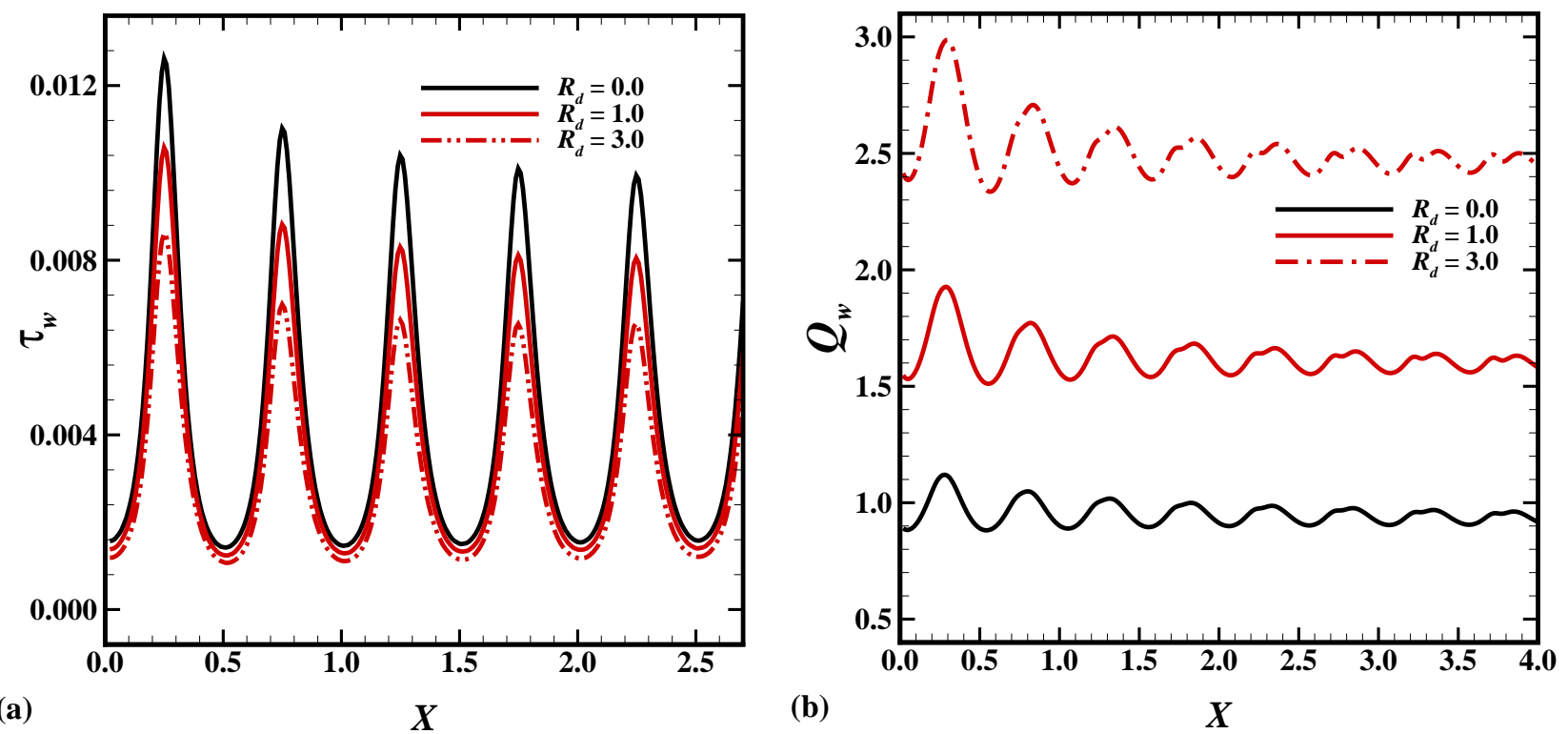

Fig. 4(a) Skin friction and (b) Rate of heat transfer for $R_{d}=0.0,1.0,3.0$ while $\operatorname{Pr}=7.0, D_{\rho}=10.0, \gamma=0.1, \alpha_{d}=0.1, \lambda=1.0, \Theta_{w}=1.1$ and $a=0.5$.
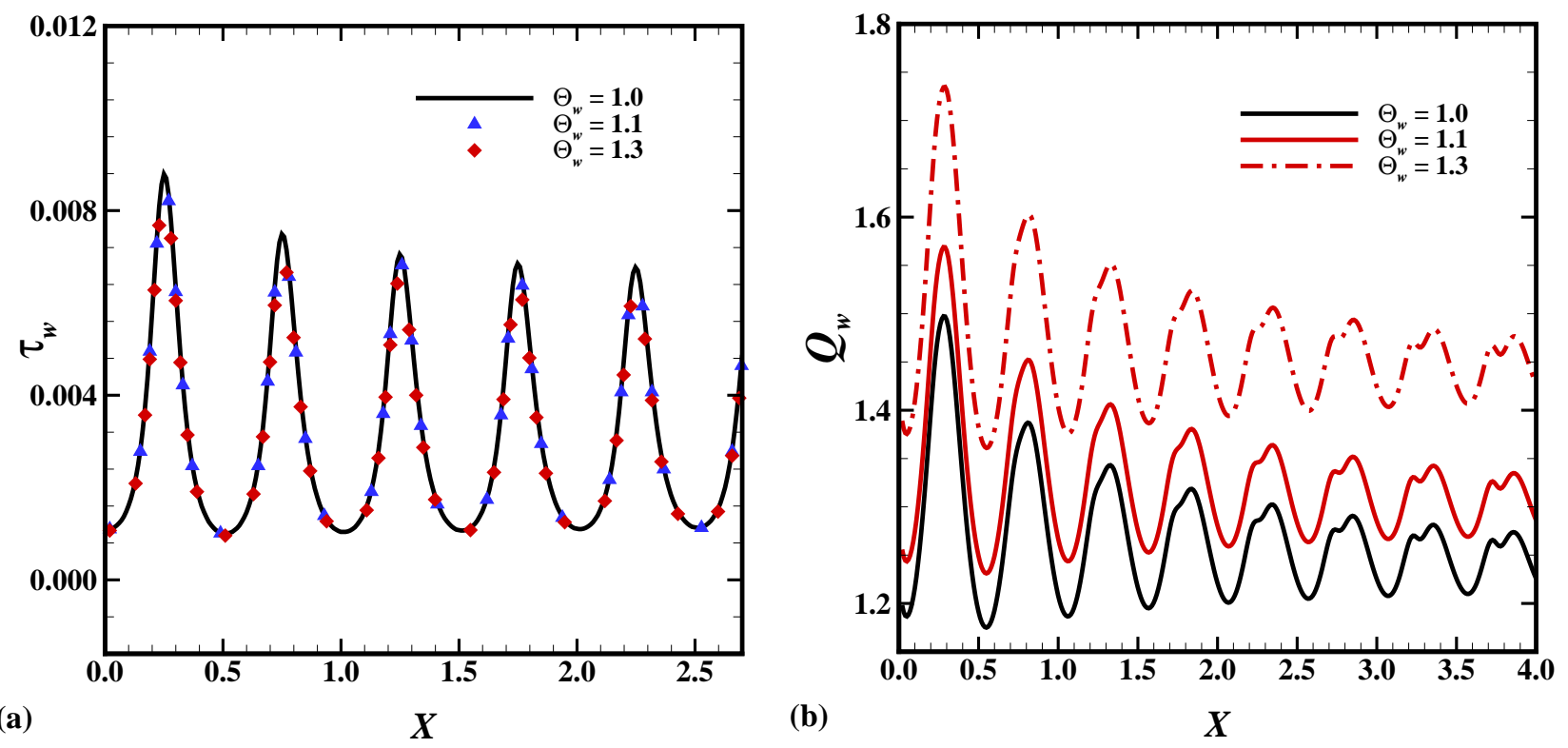

Fig. 5(a) Skin friction and (b) Rate of heat transfer for $\Theta_{w}=1.0,1.1,1.3$ while $\operatorname{Pr}=7.0, D_{\rho}=10.0, \gamma=0.1, \alpha_{d}=0.1, \lambda=1.0, R_{d}=0.5$ and $a=0.3$. 

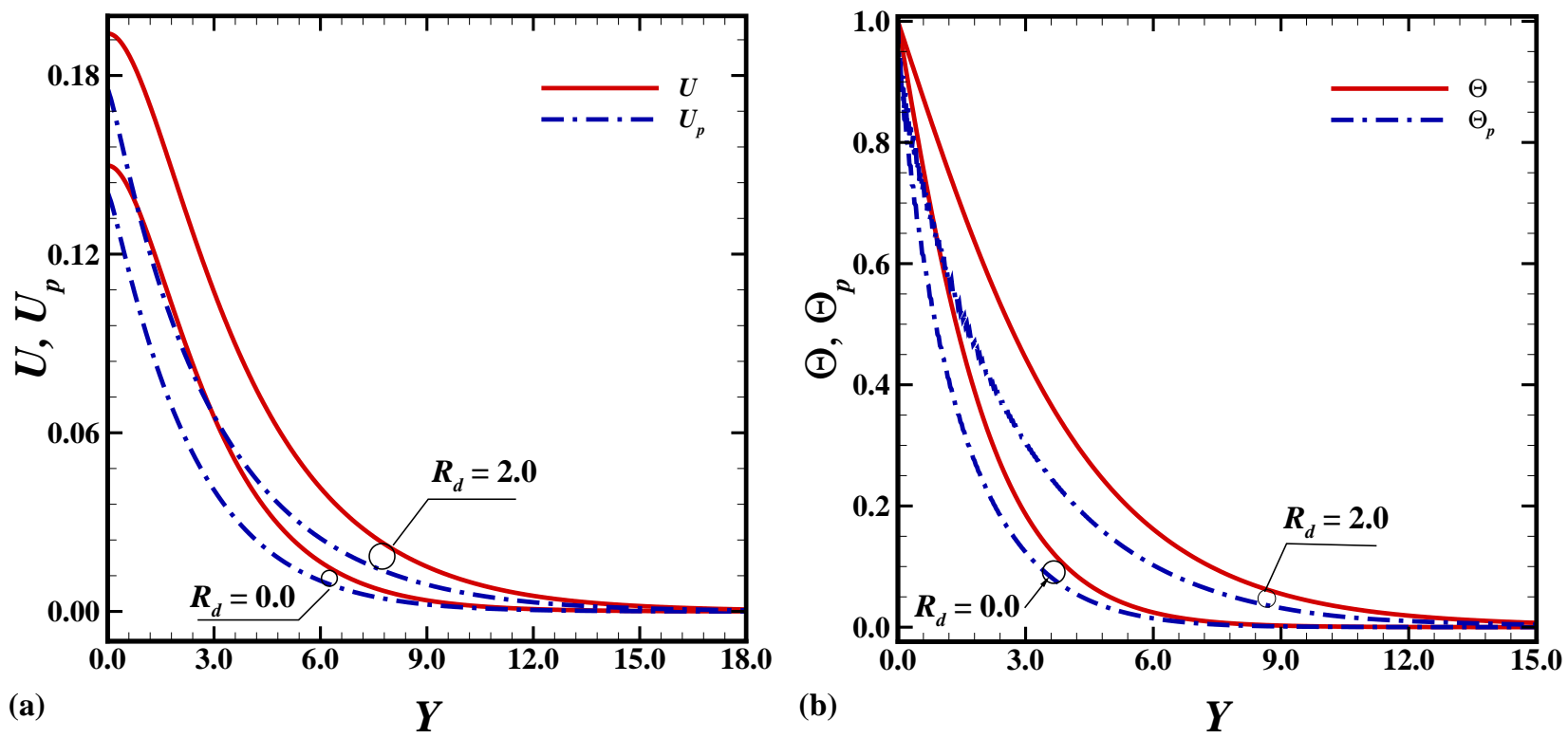

Fig. 6(a) Velocity profiles and (b) Temperature profiles for $R_{d}=0.0,2.0$ while $\operatorname{Pr}=7.0, D_{\rho}=10.0, \gamma=0.1, \alpha_{d}=0.1, \lambda=1.0, a=0.3$ and $\Theta_{w}=1.1$.
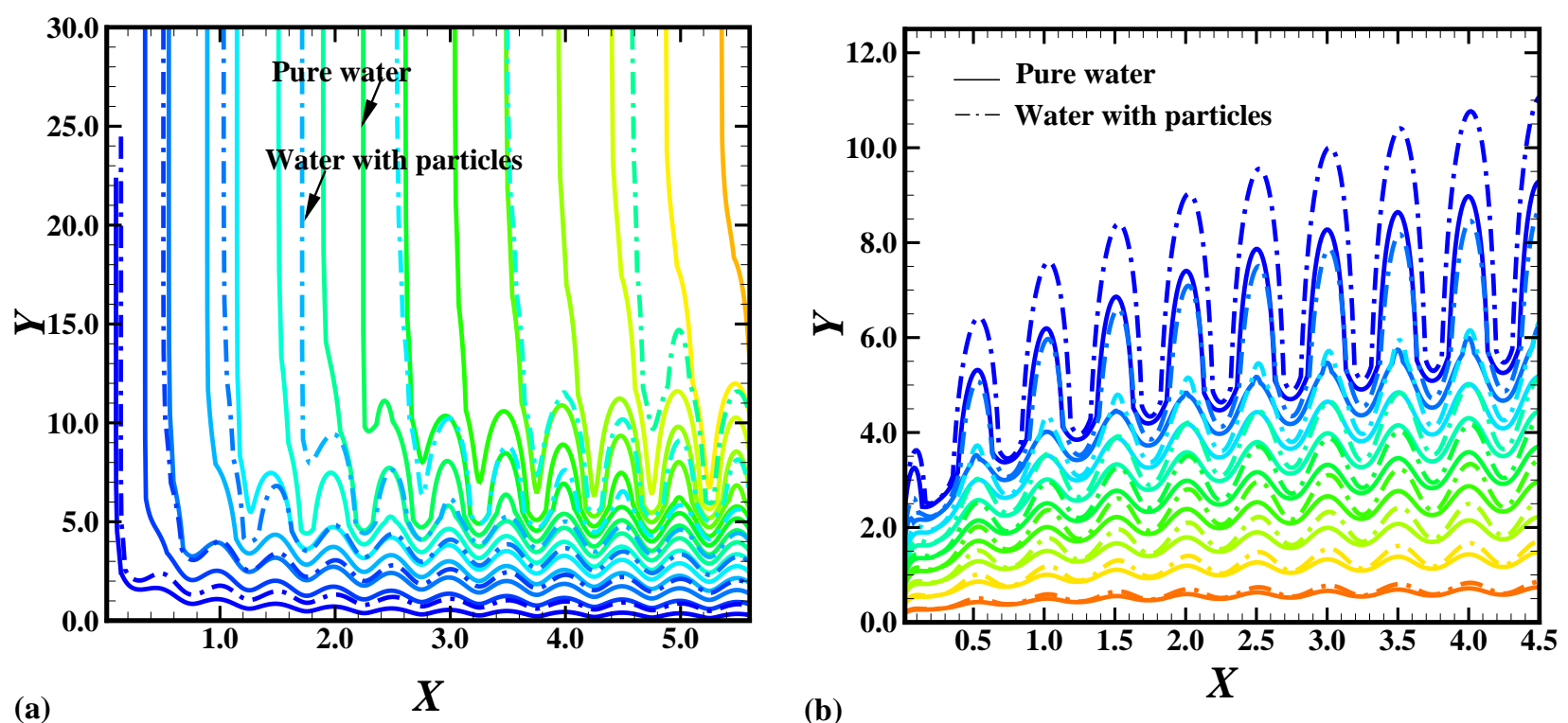

Fig. 7(a) Streamlines and (b) Isotherms for $D_{\rho}=0.0,10.0$ while $\operatorname{Pr}=7.0$, $\gamma=0.1, \alpha_{d}=0.1, \lambda=1.0, R_{d}=2.0, \Theta_{w}=1.1$ and $a=0.3$. 